\title{
FORMULATION AND EVALUATION OF PERINDOPRIL MICROENCAPSULES USING DIFFERENT POLYMER
}

\author{
SHAJAN ABRAHAM ${ }^{1}$, ABHILASH TV ${ }^{2}$, LEENA JACOB ${ }^{1 *}$ \\ ${ }^{1}$ Department of Pharmaceutics, Nazareth College of Pharmacy, Thiruvalla, Kerala, India. ${ }^{2}$ Department of Pharmaceutics, Shredevi College \\ of Pharmacy, Mangalore, Karnataka, India. Email: leenajacob789@gmail.com \\ Received: 26 August 2016, Revised and Accepted: 02 November 2016
}

\section{ABSTRACT}

Objective: The study was conducted with an objective to achieve a potential sustained release oral drug delivery system of an antihypertensive drug, perindopril which is a angiotensin converting enzyme inhibitor having half-life of 2 hrs. Perindopril is water-soluble drug, so we can control or delay the release rate of drug using release retarding polymers. This may also decrease the toxic side effects by preventing the high initial concentration in the blood.

Methods: Microcapsules were prepared by solvent evaporation technique using Eudragit L100 and ethyl cellulose as a retarding agent to control the release rate and magnesium stearate as an inert dispersing carrier to decrease the interfacial tension between lipophilic and hydrophilic phase.

Results: Prepared microcapsules were evaluated for the particle size, percentage yield, drug entrapment efficiency, flow property, and in vitro drug release for $12 \mathrm{hrs}$. Results indicated that the percentage yield, mean particle size, drug entrapment efficiency, and the micrometric properties of the microcapsules were influenced by various drug:polymer ratio. The release rate of microcapsules could be controlled as desired by adjusting the combination ratio of dispersing agents to retarding agents.

Conclusion: Perindopril microcapsules can be successfully designed to develop sustained drug delivery that reduces the dosing frequency and their by one can increase the patient compliance.

Keywords: Perindopril, Microencapsules, Solvent evaporation.

(C) 2017 The Authors. Published by Innovare Academic Sciences Pvt Ltd. This is an open access article under the CC BY license (http://creativecommons. org/licenses/by/4. 0/) DOI: http://dx.doi.org/10.22159/ajpcr.2017.v10i2.14899

\section{INTRODUCTION}

Conventional oral dosage forms provide specific drug concentration in systemic circulation without offering any control over drug delivery and also cause great fluctuations in plasma levels. In case of conventional therapy, drug administered not only interact with targeted cells but also with normal healthy cells which often results in toxic effects, and this therapy also includes frequent administration of therapeutic agent which reduces patient compliance. The therapy also involves administration of high concentration of therapeutic agent to maintain its effect [1,2]. Research is gaining much attention is "controlled drug delivery" because of their more precise, reduced toxicity, and enhanced efficacy [3]. Various approaches in delivering a therapeutic substance to the target site in a sustained or controlled release fashion. In this regard, novel drug delivery systems have many benefits which include improved therapy by increasing the efficacy and duration of drug activity, increased patient compliance through decreased dosing frequency and convenient routes of administration and improved site specific delivery to reduce unwanted adverse effects $[4,5]$.

The most exciting opportunities in CDDS lies in the area of responsive delivery systems, with which it will be possible to deliver drugs through implantable devices in response to a measured blood level or to deliver a drug precisely to a targeted site. Much of the developments of novel materials in controlled drug delivery are focusing on the preparation and use of the responsive polymers with specifically defined macroscopic and microscopic structural and chemical features. One such approach is using polymeric microcapsules as carriers for drug. Microencapsulation for oral use has been employed to control the drug release, and to reduce or eliminate gastrointestinal tract irritation. In addition, multi particulate delivery systems spread out more uniformly in the gastrointestinal tract. This result in more reproducible drug absorption and reduces local irritation when compared to single unit dosage forms.

Perindopril belongs to a class of medicines called angiotensin converting enzyme inhibitors, or ACE inhibitors. Since the drug is preferentially absorbed in the proximal small intestine (narrow absorption window), the drug displays oral bioavailability problems in conventional dosage forms. An elegant and simple way to improve drug absorption and release the drug in controlled manners is to hold the drug delivery system above the absorption window. As oral administration of perindopril results in peak plasma concentrations that occur at approximately $1 \mathrm{hr}$, half-life is $1.2 \mathrm{hrs}$. The absolute oral bioavailability of perindopril is about $75 \%$. Following absorption, approximately $30-50 \%$ of systemically available. Perindopril doses of 4-16 mg administered once daily are more effective than placebo in the treatment of mild-to-moderate hypertension $[6,7]$.

The solvent evaporation method of microencapsulation involves the use of emulsification of a solution containing polymer and drug with an additional medium in which the drug and polymer cannot dissolve [8].

The aim of this work was to develop microcapsules of perindopril by solvent evaporation technique. Perindopril is water-soluble drug, we can control or delay the release rate of drug using microencapsulation. This may also decrease the toxic side effects by preventing the high initial concentration in the blood. Due to physicochemical properties and short half-life, it is a good candidate for microencapsulation.

\section{METHODS}

Perindopril was obtained as a gift sample from the Glenmark Pvt. Ltd., Goa, India. Copolymers (Eudragit ${ }^{\circledR}$ L-100 and S-100) and ethyl 
cellulose were supplied as gift sample by Yarrow Chem. India Ltd., heavy liquid paraffin, was obtained from Nice Chemicals, Kochi, Kerala, India. Acetone was supplied by Labort Fine Chem, Surat, India. $n$-Hexane was supplied by Merck Specialties, Mumbai, India. Magnesium stearate was obtained from National Chemicals, Gujarat, India. All other chemicals and reagent used in this study were of analytical grade (Table 1).

\section{Method of preparation [9]}

Perindopril microspheres were prepared by solvent evaporation method using different quantities of Eudragit L100 and ethyl cellulose polymers. Approximately, $0.5,1.0$, and $1.5 \mathrm{~g}$ were accurately weighed and dissolved in $13.5 \mathrm{ml}$ of acetone with stirring. A $500 \mathrm{mg}$ quantity of drug and $50 \mathrm{mg}$ of magnesium stearate were then dispersed in the polymer solution. The resultant milky white dispersion was poured into a vessel containing a mixture of $135 \mathrm{ml}$ of liquid paraffin and $15 \mathrm{ml}$ of $n$-hexane and stirred for 5 hrs using a homogenizer fitted with a fourblade "butterfly" propeller. Stirring was continued for $3 \mathrm{hrs}$ at $1000 \mathrm{rpm}$ or until the acetone was completely evaporated. Following removal of acetone, the resultant microcapsules were harvested by vacuum filtration after which they were washed 4 times with $25 \mathrm{ml}$ of $n$-hexane and dried at room temperature $\left(250^{\circ} \mathrm{C}\right)$ for $24 \mathrm{hrs}$ microcapsules with drug/polymer.

\section{Evaluation parameters}

\section{Appearance}

The general appearance and elegance of microcapsule were identified visually, which include size, shape, color, presence or absence of an odor, taste, and surface texture.

\section{Percentage yield}

The measured weight was divided by total amount of all nonvolatile components which were used for the preparation of microcapsule.

Percentage yield $(\%)=($ Weight of microcapsules/Total weight of excipient and drug) $\times 100$

\section{Drug entrapment efficiency}

The amount of perindopril present in the microcapsules is determined by extracting into acid buffer. Microcapsules are crushed and powdered using a mortar and pestle and accurately weighed amount of this powder is extracted into $100 \mathrm{ml}$ of acid buffer $(0.1 \mathrm{M}$ hydrochloric acid) by stirring at $1000 \mathrm{rpm} 2 \mathrm{hrs}$. The solution is filtered; suitable dilutions are made and absorbance was measured at $212 \mathrm{~nm}$ using ultraviolet (UV) spectrophotometer.

Drug entrapment efficiency (\%) = Experimental drug content/ Theoretical drug content $\times 100$

\section{Micrometric properties [9]}

\section{Particle size}

Determination of average particle size of perindopril microencapsules was carried out by the optical microscopy method. A minute quantity of microcapsules dispersed in glycerin and then spread on clean glass slide and average size of 100 microcapsules were determined in each batch.

\section{Angle of repose}

Determination of angle of repose perindopril microencapsules was carried out by employing fixed funnel method:

Angle of repose, $\theta=\tan ^{-1}(\mathrm{~h} / \mathrm{r})$

Where, $\theta=$ Angle of repose,

$\mathrm{h}=$ Height of the pile,

$r=$ Radius of the base of the pile.

\section{Scanning electron microscopy (SEM) [9]}

The samples for SEM analysis were prepared by following method. The shape and surface morphology of microcapsules were studied using SEM. Microcapsules were mounted directly onto the SEM sample stub using double-sided sticking tape and coated with gold film (thickness $200 \mathrm{~nm}$ ) under reduced pressure. The microcapsules were viewed at an accelerating voltage of $10 \mathrm{KV}$.

\section{Drug release study [10]}

In vitro release study: In vitro dissolution profile of each formulation was determined by employing rotating basket apparatus (Apparatus I) method $\left(900 \mathrm{ml}\right.$ of $\left.0.1 \mathrm{~N} \mathrm{HCl}, 100 \mathrm{rpm}, 37 \pm 0.5^{\circ} \mathrm{C}\right)$. Microcapsules equivalent to $100 \mathrm{mg}$ of perindopril was loaded into the dissolution apparatus. Aliquot of $5 \mathrm{ml}$ was withdrawn from the dissolution medium at suitable time intervals; sink conditions are maintained to keep the total volume constant. The dug release at different time intervals was measured using a double beam UV spectrophotometer at $212 \mathrm{~nm}$. Results of in vitro drug release studies obtained from absorbance data were tabulated and shown graphically as cumulative \% drug released versus time. The study was conducted in triplicate.

\section{Stability study $[11,12]$}

Stability testing was carried out to investigate the degradation of drug from microcapsule during storage condition. Best two (F3, F4) of the optimized perindopril microcapsule formulation sealed in glass vial and stored in accelerated condition $\left(400 \pm 20^{\circ} \mathrm{C} / 75 \% \pm 5 \% \mathrm{RH}\right)$ for 6 months. Protection from effect of change in the relative humidity was provided by keeping the formulation in the stability chamber. Samples from each batch were withdrawn after definite time intervals, drug entrapment efficiency, and drug release were analyzed.

\section{RESULTS}

\section{Formulation characteristics}

Perindopril microencapsules were prepared by solvent evaporation method using two different polymers Eudragit L100 and ethyl cellulose. The sustained release polymers Eudragit L100 and ethyl cellulose were employed to bind the inert solid dispersing carrier into microsphere and control the drug release rate. The popular method for the encapsulation of drugs within water-insoluble polymers is the emulsion solvent evaporation method. This technique offers several advantage and is preferable to other preparation methods such as spray drying, sonication, and homogenization because it requires only mild conditions such as ambient temperature and constant stirring. The use of magnesium stearate as a dispersion agent decreased the interfacial tension between the lipophilic and hydrophilic phases of the emulsion and further simplified the formation of microcapsules. As the solvent evaporated, the viscosity of the individual droplets increased,

Table 1: Formulation of microcapsules

\begin{tabular}{llllll}
\hline Formulation code & Drug $(\mathbf{m g})$ & Eudragit L100 $(\mathbf{m g})$ & Ethyl cellulose $\mathbf{( m g})$ & Magnesium stearate $(\mathbf{m g})$ & Acetone $(\mathbf{m l})$ \\
\hline F1 (1:1) & 500 & 500 & - & 50 & 13.5 \\
F2 (1:2) & 500 & 1000 & - & 50 & 13.5 \\
F3 (1:3) & 500 & 1500 & - & 50 & 13.5 \\
F4 (1:1) & 500 & - & 500 & 50 & 13.5 \\
F5 (1:2) & 500 & - & 1000 & 50 & 13.5 \\
F6 (1:3) & 500 & - & 1500 & 50 & 13.5 \\
\hline
\end{tabular}


and highly viscous droplets were observed to coalesce at a faster rate than they could be separated. Magnesium stearate formed a thin film around the droplets and thereby reduced the extent of coalescence, before hardening of the capsules, on collision of droplets. The resultant microcapsules were free-flowing, and the use of magnesium stearate was deemed effective. Liquid paraffin was selected as a bulk or outer phase since drug and polymers are only very slightly soluble in liquid paraffin. Acetone has a dielectric constant of 20.7 and was therefore chosen as the dispersed or inner phase since solvents with dielectric constants between 10 and 40 show poor miscibility with liquid paraffin. Microcapsules that were formulated with low concentrations of Eudragit L100 and ethyl cellulose were irregular, nonspherical, soft, and had poor flow ability, therefore, they were not considered suitable for analysis. During processing, it was observed that stirrer speeds of $<500 \mathrm{rpm}$ were not sufficient to produce microcapsules, and a huge coalesce was obtained. This is due in part to inadequate agitation of the media to disperse the inner phase in discreet droplets within the bulk phase. At stirring speeds above $1000 \mathrm{rpm}$, the turbulence caused frothing and adhesion of the micro particles to the container walls and propeller blade surfaces, resulting in high shears, and smaller size of dispersed droplets. Spherical microspheres were obtained at a stirring rate of $1000 \mathrm{rpm}$; therefore, this speed was used during manufacture of all microcapsules. Many formulations were prepared, to investigate the effect of increasing amount of the polymer and dispersing agent on the microcapsule formulation. The drug polymer and drug dispersing agent ratio was altered while the amounts of solvent and stirring rate were kept constant.

\section{Yield of microcapsules}

From Table 2, it was observed that F1 showed the greatest percentage yield that is $76.40 \%$. The other formulations showed comparatively less percentage yield.

\section{Incorporation efficiency}

The values of drug entrapment efficiency are shown in Table 2. As the polymer concentration was increased, the percentage drug entrapment efficiency was increased due to increase in the viscosity of the solution. This can be attributed to the permeation characteristics of each polymer used.

\section{Micromeritic properties}

The arithmetic mean particle size of the formulations was determined by the optical microscope fitted with an ocular micrometer and stage micrometer. The average mean particle sizes of six formulations were $\quad 265.50 \pm 0.69, \quad 312.82 \pm 0.90, \quad 365.60 \pm 0.67, \quad 309.10 \pm 0.15$, $351.35 \pm 0.78$, and $323.08 \pm 0.50$, respectively. The mean particle size of the microcapsules significantly increased with increase in polymer concentration due to high viscosity of medium at a higher polymer concentration resulting in enhanced interfacial tension and diminished shearing efficiency.
Angle of repose of microcapsules was in range of $18^{\circ} 80^{\prime \prime} \pm 3.89$ to $26^{\circ}$ $56^{\prime \prime} \pm 1.17$ (Table 3 ). These values indicate an overall good free flowing nature of microcapsules of all batches.

\section{SEM}

SEM of the formulation F3 and F4 was carried out. It was observed that the microcapsules were spherical in shape with smooth surface. Photomicrograph of the formulation F3 and F4 also revealed that the microcapsules were spherical in shape (Fig. 1). In F1, the drug to polymer ratio was 1:1. So proper coating was not observed. When the concentration of polymer was increased as in F3 (1:3), proper coating was done and prepared microcapsules had the spherical shape and smooth surface.

\section{In-vitro release studies}

The ultimate aim of this work was to develop sustained release drug delivery system of perindopril. The drug release from batches F1 to F6 indicated that as the polymer concentration increased the release rate decreased (Fig. 3). The release rate of perindopril from the microcapsules can be modulated with adjusting the drug to polymer ratio. As the concentration of polymer was increased, the amount of drug release was decreased. Hence, polymer acts like a retarding material. It is important to note that the dissolution behavior of granules and powders is greatly influenced by their wettability, surface area, and particle size distribution. The formulation F3 shows the maximum cumulative drug release within $12 \mathrm{hrs}$. The overall cumulative \% release for F1, F2, F3, F4, F5, and F6 were found to be $84.20 \pm 0.66$, $74.43 \pm 1.69,94.58 \pm 0.79,89.60 \pm 1.92,81.82 \pm 0.53$, and $78.87 \pm 1.30$ at the end of $12^{\text {th }} \mathrm{hr}$.

Table 2: Yield of microcapsules

\begin{tabular}{lll}
\hline Formulation code & Percentage yield & $\begin{array}{l}\text { \% Drug entrapment } \\
\text { efficiency }\end{array}$ \\
\hline F1 & $76.40 \pm 3.04$ & $75.20 \pm 0.50$ \\
F2 & $73.09 \pm 2.05$ & $80.10 \pm 2.79$ \\
F3 & $68.90 \pm 1.50$ & $85.10 \pm 1.05$ \\
F4 & $74.07 \pm 2.35$ & $77.25 \pm 1.20$ \\
F5 & $72.65 \pm 1.67$ & $82.75 \pm 1.56$ \\
F6 & $65.25 \pm 1.35$ & $87.40 \pm 0.75$ \\
\hline
\end{tabular}

Table 3: Micromeritic properties

\begin{tabular}{lll}
\hline Formulation code & Mean particle size $(\boldsymbol{\mu m})$ & Angle of repose \\
\hline F1 & $265.50 \pm 0.69$ & $18^{\circ} 80^{\prime \prime} \pm 3.89$ \\
F2 & $312.82 \pm 0.90$ & $21^{\circ} 39^{\prime \prime} \pm 2.78$ \\
F3 & $365.60 \pm 0.67$ & $22^{\circ} 44^{\prime \prime} \pm 1.93$ \\
F4 & $309.10 \pm 0.15$ & $25^{\circ} 64^{\prime \prime} \pm 0.68$ \\
F5 & $351.35 \pm 0.78$ & $22^{\circ} 76^{\prime \prime} \pm 3.39$ \\
F6 & $323.08 \pm 0.50$ & $26^{\circ} 56^{\prime \prime} \pm 1.17$ \\
\hline
\end{tabular}

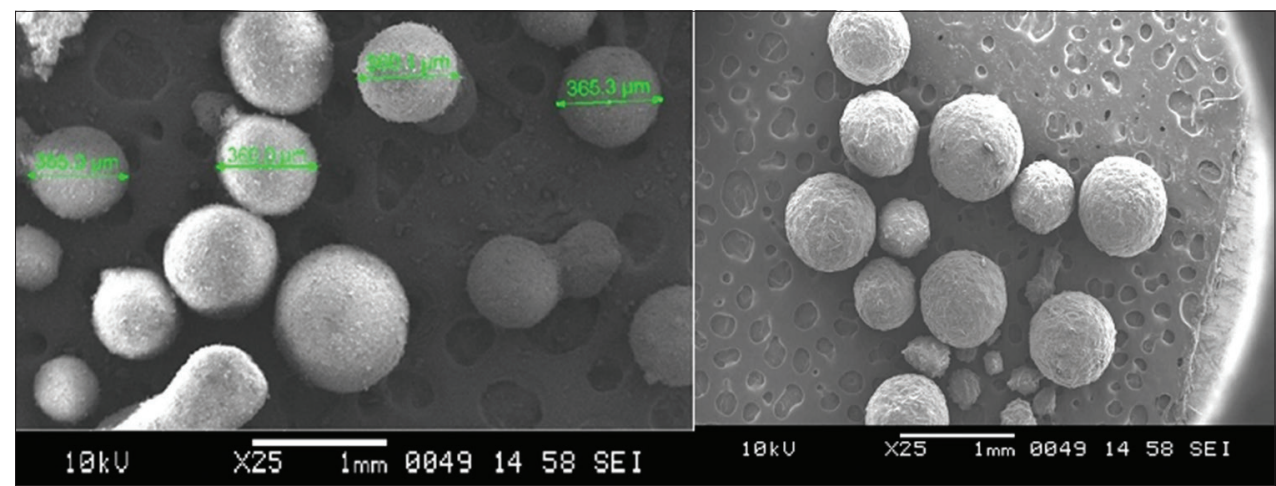

Fig. 1: Scanning electron microscopy F3, F4 


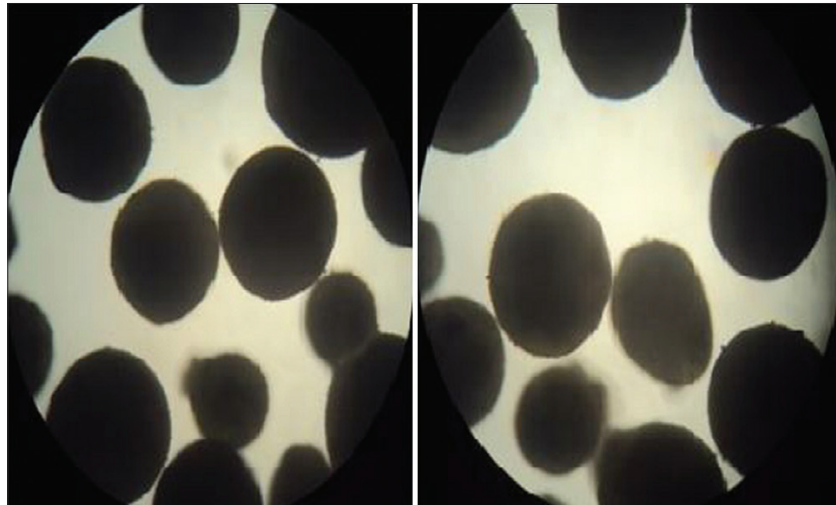

Fig. 2: Microscopic evaluation F3

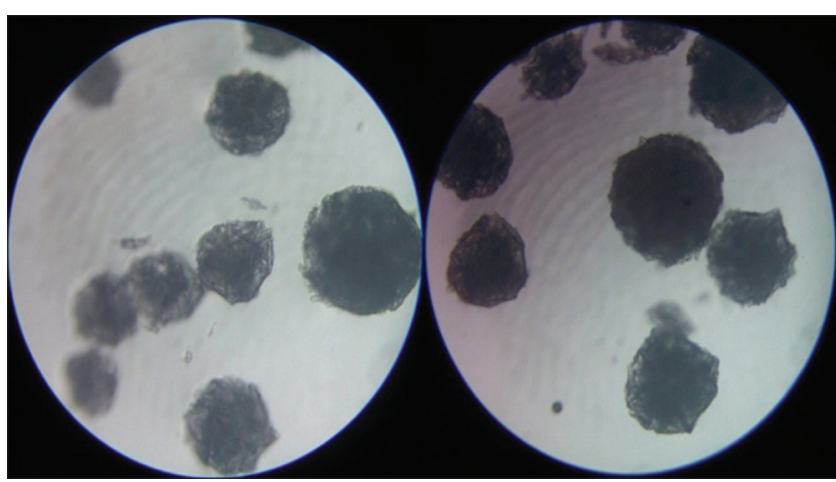

Fig. 3: Microscopic evaluation of F4

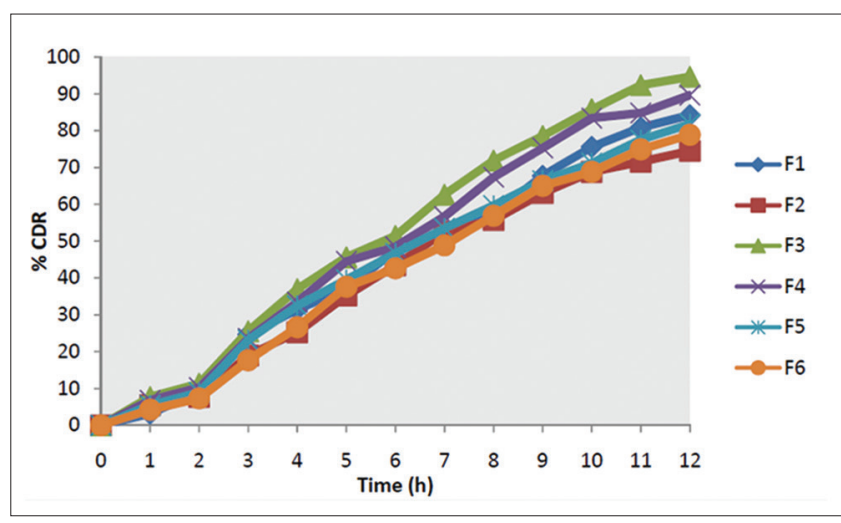

Fig. 4: In vitro release studies

\section{CONCLUSION}

This study was attempted to develop microcapsules of perindopril for the treatment of blood pressure. Sustained release microcapsules of perindopril were developed using combination of release retardant polymers, Eudragit L100, and ethyl cellulose by solvent evaporation method. Six formulations were prepared using two different polymers. All the formulations were subjected to drug entrapment studies, particle size determination, and in vitro drug release studies. The microcapsules were satisfactory considering their percentage yield, size, and drug entrapment. On the basis of release studies, it was indicated that the polymers Eudragit L100 and ethyl cellulose retards the release of perindopril from microcapsules. The preparation process was simple, reproducible, and the resultant microcapsules had the desired micrometric properties. Hence, this study revealed that the microcapsules of perindopril prepared by solvent evaporation method could sustain the drug release, and thus, it is a relevant alternative of conventional dosage forms.

\section{REFERENCES}

1. Jain NK. Controlled and Novel Drug Delivery. $5^{\text {th }}$ ed. New Delhi: CBS Publishers and Distributers; 2002. p. 237-51.

2. Venkatesan P, Manavalan R, Valliappan K. Microencapsulation: A vital technique in novel drug delivery system. J Pharm Sci Res 2009;1(4):26-35.

3. Hoffman A, Stepensky D, Lavy E, Eyal S, Klausner E, Friedman M. Pharmacokinetic and pharmacodynamic aspects of gastroretentive dosage forms. Int J Pharm 2004;277(1-2):141-53.

4. Brahmankar DM, Jaiswal SB. 4. Atriatse. Biopharmaceutics and Pharmacokinetics. $3^{\text {rd }}$ ed. New Delhi: Vallabh Prakashan; 2000. p. 335-71.

5. Ma N, Xu L, Wang Q, Zhang X, Zhang W, Li Y, et al. Development and evaluation of new sustained-release floating microspheres. Int J Pharm 2008;358(1-2):82-90.

6. Pharma Professional Services; 2010. Available from: http://www.drug. infosys.com/Drug.aspx/drugCode961/perindoprilerbbu mine 24/3/10. [Last cited on 2012 Jun 28].

7. Wikimedia Foundation; 2013. Available from: http://www.en.wikipedia. org/wiki/Perindopril. [Last cited on 2013 Feb 27].

8. Horoz BB, Kiliic M, Arslan N, Baykara YT. Effect of different dispersing agents on the characteristics of Eudragit microspheres prepared by a solvent evaporation method. J Microencapsul 2004;21(2):191-202.

9. Saravanan M, Dhanaraju MD, Sridhar SK, Ramachandran S, Sam SK, Anand $\mathrm{P}$, et al. Preparation, characterization and in vitro release kinetics of ibuprofen polystyrene microspheres. Indian J Pharm Sci 2004;66(3):287-92.

10. Patel A, Ray S, Thakur RS. In vitro evaluation and optimization of controlled release floating drug delivery system of metformin hydrochloride. DARU 2006;14(2):57-64

11. Lachman L, Partic D. Kinetic principles and stability. Theory and Practice of Industrial Pharmacy. $3^{\text {rd }}$ ed. Philadelphia, PA: Varghese Publishing House; 1987. p. 767.

12. Kulkarni GT, Gowthamarajan K, Suresh B. Stability testing of pharmaceutical products: An overview. Indian J Pharm Educ 2004;38(4):194-202. 